\title{
Hermansky-Pudlak syndrome
}

INSERM

\section{Source}

INSERM. (1999). Orphanet: an online rare disease and orphan drug data base.

Hermansky-Pudlak syndrome. ORPHA:79430

Hermansky-Pudlak syndrome (HSP) is a multi-system disorder characterized by oculocutaneous albinism, bleeding diathesis and, in some cases, neutropenia, pulmonary fibrosis, or granulomatous colitis. HPS comprises eight known disorders (HPS-1 to HPS8), the majority of which present with the same clinical phenotype to varying degrees of severity. 